\title{
INVESTIGATION OF THE SURFACE RESISTIVITY TOLERANCE OF THE KICKER CERAMIC VACUUM CHAMBER AT APS*
}

\author{
C. Doose ${ }^{\dagger}$, L. Emery, and S. H. Kim \\ Advanced Photon Source, Argonne National Laboratory, Argonne, IL
}

\begin{abstract}
The conductive coating on kicker ceramic vacuum chambers was found to affect the pulse width, amplitude, and phase of the kicker magnetic field. Differences in the surface resistivity between the chambers caused each kicker magnetic field to have a slightly different pulse shape, resulting in unwanted betatron oscillations of the stored beam during injection into the storage ring at the Advanced Photon Source. Orbit oscillations were measured with the present kicker mismatch and optimized for a closed bump. Tracking simulations of the closed bump provided an idealized tolerance for the variation of chamber surface resistivity consistent with minimizing the betatron oscillations. The pulse shape of the kicker current was dependent on the current level and the coupling between the kicker magnet and chamber. Local magnetic fields in two prototype kicker chambers were measured and compared with eddy current calculations.
\end{abstract}

\section{INTRODUCTION}

The Advanced Photon Source Storage Ring (SR) uses ceramic vacuum chambers in the sections of four pulsed kicker magnets for beam injection. The inner surfaces of the ceramic chambers are coated with a conductive material. The coating must conduct beam image currents while allowing the kicker magnetic field to penetrate the chamber wall. Differences in the coating surface resistivity, $R$, between the chambers caused each kicker magnetic field to have mismatched pulse shapes, resulting in unwanted betatron oscillations of the stored beam during injection.

The nominal coating $R_{s}$ was originally chosen to be 0.1 $\Omega$, with a tolerance of $20 \%$, as a compromise between kicker field attenuation and image current power dissipation [1]. Recent SR operations in a top-up mode require beam injection every 2 minutes in order to maintain a constant average beam current.

This study investigates the present betatron oscillations in the SR at the light source points and defines acceptance criteria for the chamber coating consistent with minimizing these oscillations. Tracking simulation results specified a $2 \%$ tolerance of the coating $R_{s}$ to achieve the desired rms betatron oscillation amplitude of $35 \mu \mathrm{m}$. This study also measures pulse shapes of the kicker current and magnetic field with and without chambers of varying $R_{s}$. These measurements were taken to determine the best operating conditions given the present chamber and power supply variations and to specify future improvements.

\footnotetext{
*Work supported by U.S. Department of Energy, Office of Basic Energy Sciences under Contract No. W-31-109-ENG-38.

‘doose@aps.anl.gov
}

\section{BEAM MOTION MEASUREMENT}

At the optimized settings for a closed bump the current for the four injection kickers were measured using an oscilloscope. The current pulse shapes were not identical to each other because of current-dependent power supply differences, especially in the decay portion. The highercurrent kickers have a longer tail, therefore the betatron oscillation measured included the effect of the power supply and the chamber $R_{s}$ differences. The power supply effects alone could not be characterized using a single kicker because at currents above $40 \%$ the beam was lost.

We filled the SR with a single bunch, and adjusted the current amplitudes of the four kickers for a closed bump (i.e., no betatron oscillation). Letting the kicker pulse continuously at $2 \mathrm{~Hz}$, we varied the timing of the injection trigger to effectively change the timing of the peak of the kicker current pulse relative to the bunch. We measured the rms amplitude of the betatron oscillation by taking history data from a beam position monitor close to a light source point. The closed bump was optimized and data taken with the sextupole power supplies off and on in the injection sectors containing the four kickers. This gave a measure of how the kicker mismatch compares with nonlinear effects of the sextupoles, and is shown in Fig. 1.

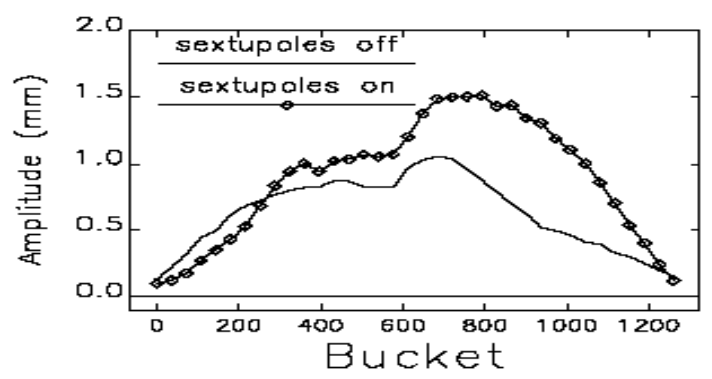

Figure 1: Present rms orbit oscillation amplitude at a light source point as a function of buckets around the SR.

\section{SPECIFICATIONS}

The betatron oscillation created by the mismatch in the kicker field shape decohered in about 20 turns $(70 \mu \mathrm{s})$, but the emittance blow-up that followed lasted two damping times $(20 \mathrm{~ms})$. These are short periods relative to the injection interval, and any small disturbance would seem to contribute little to the average rms beam motion. The specifications of maximum allowed orbit motion or emittance blow-up for these periods are difficult to obtain from synchrotron light users because they run experiments in a variety of time scales. The beam size at the insertion device source points is about $350 \mu \mathrm{m}$. We 
assume a $10 \%$ of beam size $(35 \mu \mathrm{m})$ betatron oscillation from the injection event, which, after decoherence, turns into a small emittance dilution of $0.5 \%$. (Compare this to the present large oscillation amplitude of approximately 1 $\mathrm{mm}$.)

The specification on the resistive coating tolerance was determined from the above beam motion specification and tracking simulation as follows. Because we are only interested in the waveform mismatch as a result of the variation of $R_{s}$ between kickers, we assumed identical current waveforms for all kickers. We smoothed one measured current waveform for use as a realistic model. We then applied a low-pass filter with a time constant of $0.3 \mu$ s (assuming a cylindrical chamber) to this waveform to create the magnetic field waveforms. The time constant, $\tau$, for an elliptic chamber may be expressed as

$$
\tau=f \frac{\mu_{o} a}{2 R_{s}},
$$

where the major radius $a$ was $51 \mathrm{~mm}$, with $f=1.25$ for the chambers used in this study.

The first-order effect of the low-pass filter was to shift the peak and change the amplitude of the waveform for all kickers in the simulation. These effects are normally compensated in actual SR operation by adjusting the timing and amplitudes for a closed bump for a reference bunch. For the simulation the timing and amplitude of each waveform were adjusted in the same way, and the value of $\tau$ for the highest-current kicker was varied relative to the nominal $0.3 \mu$ s for the other three kickers.

The secondary effect of $\bullet$ was to change the rise and decay slope of each kicker field, which cannot be compensated for in the actual operation of the SR, and was the cause of betatron oscillation for the non-reference bunch. The tracking simulation of a $20 \%$ variation in $R_{s}$ for the highest-current kicker chamber gave a maximum betatron oscillation of about $400 \mu \mathrm{m}$. For a $2 \%$ variation the betatron oscillation as shown in Fig. 2 is $35 \mu \mathrm{m}$, which meets our specification.

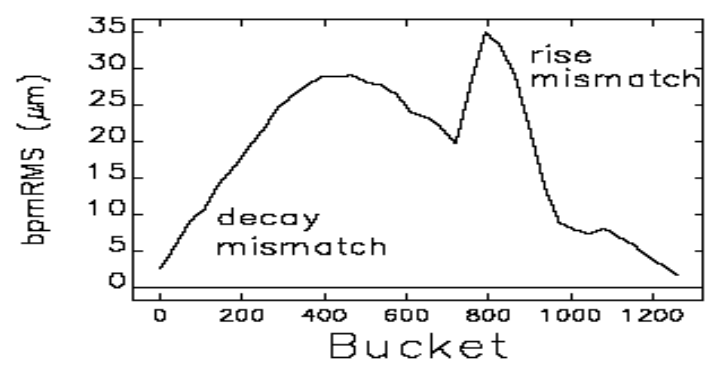

Figure 2: Tracking simulation showing rms orbit oscillation produced from a $2 \%$ variation in - of the chamber in the highest-current kicker as a function of buckets around the ring.

\section{MAGNETIC FIELD MEASUREMENTS}

Magnetic measurements were taken to confirm the power supply and chamber effects on the kicker current and magnetic field. The magnetic field in the mid-plane of a spare kicker magnet was measured with and without ceramic chambers. The kicker field uniformity without a chamber was approximately $0.1 \%$. Two prototype ceramic chambers with ceramic wall thicknesses of $3.2 \mathrm{~mm}$ and an axial length of $0.74 \mathrm{~m}$ were used. The cross-section approximates an ellipse with major and minor axes of 102 $\mathrm{mm}$ and $52 \mathrm{~mm}$, respectively. The $R_{s}$ of the chamber coating had been mapped earlier by an eddy current measurement method [2]. One chamber had an average $R_{s}$ of $0.05 \Omega$ with a variation of 0.04 to $0.07 \Omega$ along its length. The average $R_{s}$ of the other chamber was $0.34 \Omega$ with a variation from 0.14 to $0.63 \Omega$.

Magnetic field data were obtained using a digital oscilloscope and integrating the signal from a 10-turn, 10mm-diameter point coil probe mounted to a moveable stage. The coil probe was scanned along the central longitudinal axis and data were collected every $20 \mathrm{~mm}$. The field, position, and current pulses for each point were saved.

\section{CHAMBER'S EFFECT ON CURRENT AND FIELD PULSE SHAPES}

\subsection{Current Pulse Shape}

The conductive coating of the ceramic chamber was penetrated by the kicker field and acted as a shorted secondary transformer winding. This may imply that the impedance of the chamber modified the kicker magnet nominal impedance. The real and imaginary parts of the effective magnet impedance with mutual inductance $M$ between the magnet and the chamber may be expressed as $\operatorname{Re}=R o+\frac{R c(\omega M)}{R c^{2}+(\omega L c)}, X e=j \omega\left[L o-\frac{L c(\omega M)^{2}}{R c^{2}+(L c)^{2}}\right],(2)$

where $\mathrm{L}_{\mathrm{c}}$ and $\mathrm{R}_{\mathrm{c}}$ are the chamber inductance and resistance, and $L_{o}$ and $R_{o}$ are the kicker magnet inductance and resistance without a chamber.

The pulse width of the current from the kicker power supply was determined by the resonant frequency of the charging capacitors and the inductive reactance looking into the power supply cables. Figure 3 shows the measured current pulse shapes with and without the chamber having an average $R_{\mathrm{s}}$ of $0.05 \Omega$. The effect of the chamber coating decreased the effective magnet inductance and current pulse-width.

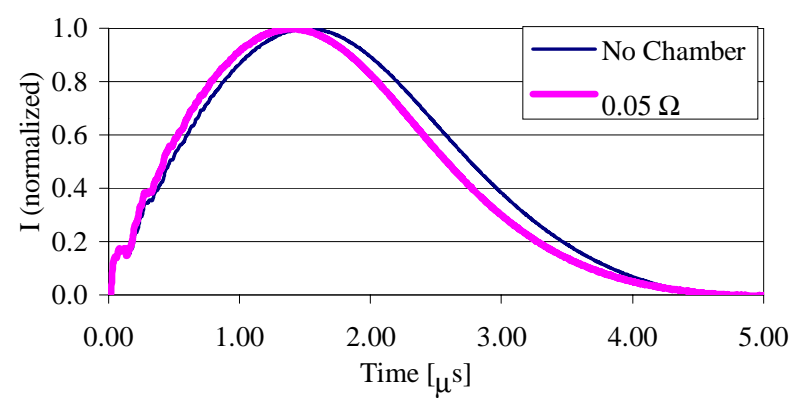

Figure 3: Kicker current pulses with and without a chamber having an average $R_{s}$ of $0.05 \Omega$. 
The current-dependent effect of the power supply on the current pulse shape is shown in Fig. 4. The lower level current pulse more closely approximates a half sine than the higher level current pulses.

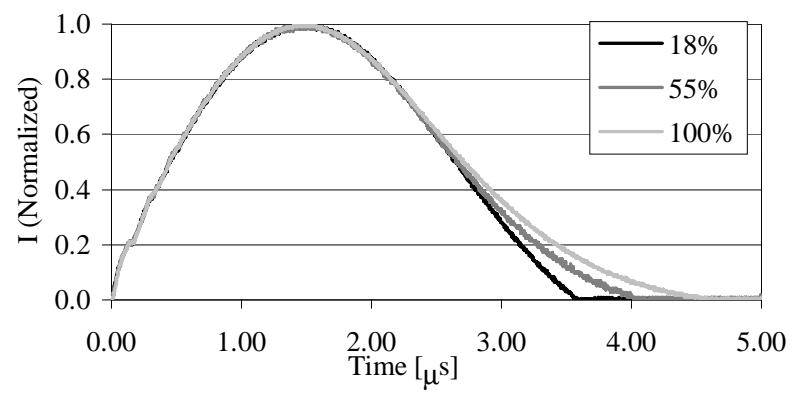

Figure 4: Current pulses normalized to their respective peaks at three current levels showing the currentdependent pulse shape. The $100 \%$ current level was approximately $3.4 \mathrm{kA}$.

\subsection{Magnetic Field Pulse Shape}

The magnetic field along the prototype chamber axis was further affected by the attenuation and phase shift of the conductive coating of the chamber. As the $R_{s}$ of the chamber coating decreased the attenuation and phase delay of the field increased. Figure 5 shows the measured attenuation and phase delay of the field at the maximum operating current $(3.4 \mathrm{kA})$ in a region of the chamber that had an $R_{s}$ of $0.05 \Omega$. The peak field in the chamber was 0.84 that of the field without the chamber, and the phase delay was $0.36 \mu \mathrm{s}$. The pulse width of the measured magnetic field without the chamber was approximately $4 \mu \mathrm{s}$; however, the shape of the driving current is not a pure half-sine pulse. The field pulse for a half-sine current pulse with $\mathrm{t}_{\mathrm{o}}=\pi / \omega$ may be expressed as

$$
\begin{aligned}
B_{i}(t) & =B_{o}\left[\frac{1}{\left(1+(\omega \tau)^{2}\right)^{1 / 2}} \sin (\omega t-\phi)+\frac{\omega \tau}{1+(\omega \tau)^{2}} e^{-t / \tau}\right], \\
& =B_{o} \frac{\omega \tau}{1+(\omega \tau)^{2}}\left(1+e^{t o / \tau}\right) e^{-t / \tau} \quad \text { for } t \geq t_{o}
\end{aligned}
$$

where $\phi=\tan ^{-1}(\omega \tau)$ and $\tau$ is expressed in Eq. (1) [3].

The relative difference in the magnetic field attenuation for a given relative difference in $R_{s}$ was larger for lower coating $R_{s}$ values. Figure 6 shows the measured and calculated attenuation magnitude vs. chamber coating $R_{s}$. The solid curve in the figure was calculated from Eq. (3), with $\mathrm{t}_{\mathrm{o}}=3 \mu \mathrm{s}$ (based on the $\pi / 2$ time of $1.5 \mu \mathrm{s}$ ). It can be seen from Figure 6 that below $0.15 \Omega$ the slope increases sharply and small changes in $R_{s}$ near $0.05 \Omega$ cause large changes in attenuation.

New chambers will be characterized and matched by performing magnetic measurements similar to the measurements of the prototype chambers.

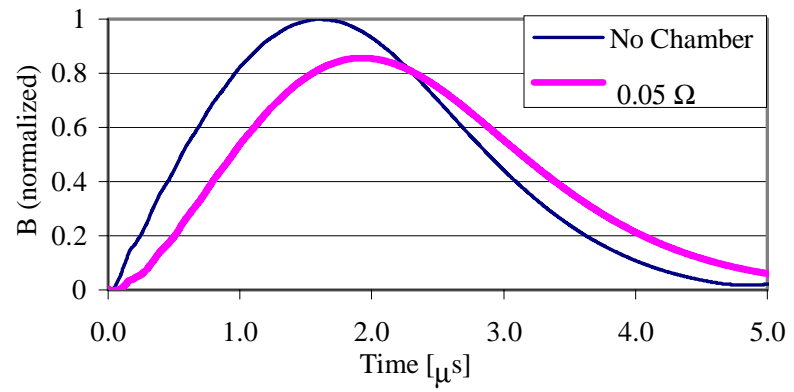

Figure 5: Measured magnetic field pulses without and with a chamber at a region with an $R_{s}$ of $0.05 \Omega$.

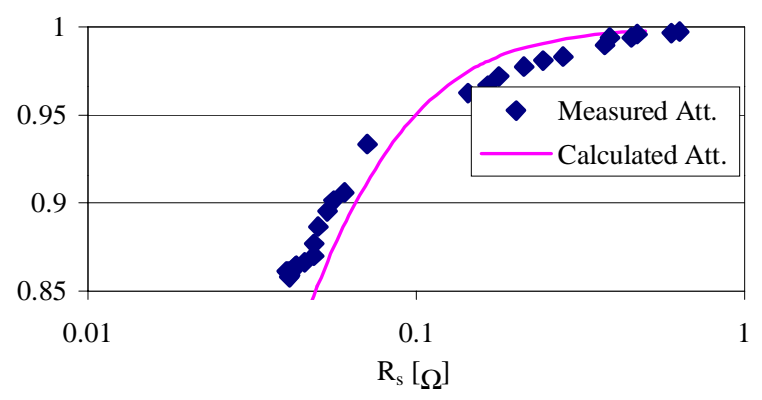

Figure 6: Magnetic field attenuation vs. chamber $R_{s}$. Measured data collected using the two chambers having non-uniform $R_{s}$ along the longitudinal chamber axis, and Eq. (3) was used for the calculated curve.

\section{CONCLUSIONS}

The measured average rms betatron oscillation in the SR using an optimized closed bump was approximately 1 $\mathrm{mm}$, due to the superposition of power supply and chamber effects. With a specified peak betatron oscillation of $35 \mu \mathrm{m}$, tracking simulations showed the need for a $2 \%$ tolerance of the $0.1 \Omega$ chamber surface resistivity. A $2 \%$ coating tolerance is rather stringent compared with the presently achievable $10 \%$ tolerance. The power supply current-dependent pulse shape was confirmed with magnetic measurements; continued work is needed to minimize this current-dependent effect. The coupling between the kicker magnet and the chamber significantly affected the power supply current pulse shape, as well as the expected field attenuation and phase delay. Matching the average surface resistivity of the chambers would minimize this problem.

\section{REFERENCES}

[1] S. Milton and D. Warner, "The APS Ceramic Chambers," Proc. EPAC94, London, pp. 2494-2496 (1994).

[2] C. Doose, K. Harkay, S. Kim, and S. Milton, "In Situ Measurement of Ceramic Chamber Conductive Coating Quality,” Proc. 1997 Particle Accelerator Conference, Vancouver, B.C., pp. 3577-3579 (1997).

[3] S.H. Kim, "Calculation of Pulsed Kicker Magnetic Field Attenuation Inside Beam Chambers," LS Note291, Argonne National Laboratory (2001). 